\begin{tabular}{ccc}
\hline & International Journal of Engineering \& Technology, $7(2.24)(2018) 474-477$ \\
SPC & International Journal of Engineering \& Technology \\
\hline
\end{tabular}

\title{
An Image Steganography using Particle Swarm Optimization and Transform Domain
}

\author{
Sanjutha.MK \\ M.Tech, Computer Science Engineering \\ School of Computing, SASTRA Deemed to be University, Thanjavur-613401 \\ *Corresponding Author E-mail: sanjuthamk30041995@gmail.com
}

\begin{abstract}
As information technology is growing tremendously, one of the major concern is information security. A technique called image steganography is used to provide better security and for safeguarding the information. In image steganography, a secret image is put into recipient image so that only the receiver and sender will be aware of the secret message. Here in this paper, a secure, optimized scheme called particle swarm optimization is used to select the pixel efficiently for embedding the secret image in to cover image. PSO(Particle Swarm Optimization) decides pixel using fitness function which is based on the cost function. Cost function calculates entropy, edge and pixels intensity to evaluate fitness. Also, a technique called discrete wavelet transform has been employed to achieve robustness and statistical undetectability. The main aim of the proposed paper is to make better security and to obtain efficient PSNR and MSE values
\end{abstract}

Keywords: Image steganography, DWT, Particle Swarm Optimization, IDWT, Security, Fitness function

\section{Introduction}

Steganography is a process of hiding a secret data which may be a text or image within other data which is nonsecret. Here only the receiver and sender will know about the existence of the message. Any steganographic method's performance will be tested based on its robustness, transparency, capacity provided and statistical undetectability. These characteristics are mostly mutually exclusive of each other. The researchers find it difficult to balance all the above factors simultaneously. However, the most two essential components which have to be given much importance are undetectability and high embedding capacity. In designing information hiding algorithms, quality of image and capacity are the key factors to be considered.

Hiding information causes some level of image distortion. But these distortions is not accepted. So to solve these problems different techniques have been proposed. Steganalysis of image steganography using wavelet-based fusion technique of high capacity is defined. Image quality metrics are also taken into consideration. First order statistics is preserved which improves the security of method against the attacks. Using SVD as steganalysis tool, the technique is compared with two other encryption techniques based on undetectability metric[1]

A cryptographic algorithm is used with steganography to achieve increased security. The file to secure is compressed, and its file is shrunk. Now compressed data is converted into cipher text using AES algorithm and put in to cover image. A genetic algorithm is put in to use for pixel assortment in the image [2].Two new steganographic techniques have been proposed in the spatial domain. LSB substitution is the basic idea that has been used. Based on previously defined conditions, bits are selected for substitution. In the second method used, hiding of one bit per pixel is done in the first method then hiding of two bit per pixel is done in second method [3]. Image steganography using QR factorization and redundant DWT is defined. Here cover selection is made by statistical texture analysis. Cover selection increases security[4].

Particle swarm optimization is population-based algorithm based on the concept of bird flocking. The exploitation and exploration characteristics are balanced by inertia weight[5].Optimal thresholding in 2-D DWT is done by using PSO. Using PSO optimal threshold set is obtained and to encode the results arithmetic coding is used [6].This paper is discussed in the following sections. Related work is explained in section 2. The proposed methodology is explained in section 3. In section 4, experimental results and analysis are explained, and section 5 contains the conclusion of the proposed system.

\section{Related Work}

Various techniques and algorithm related to image steganography have been proposed to achieve improved security. The steganographic technique consists of three layers of security, the first layer is complementation of secret message, in second layer hiding of complemented secret message in to cover image is done and finally in the third layer inverted LSB technique is used[7].To provide an efficient and novel technique for image steganography that makes secret transmission efficient using adaptive circular queue LSB substitution. The dynamic queue is used for resource distribution between the multiple recipients, and the transmission of secret data is asynchronous. RSA is used mainly for the authentication and confidentiality of secret information [8].

The combination of interpolation technique and compression technique is one of the trending topics in designing information hiding algorithms. Better image quality and higher capacity are the key factors of information hiding. Adaptive information hiding is a trending topic in network security and hiding the information[9]. Integer is mapped to an integer by wavelet transform so that original 
image can be reconstructed perfectly. Message bit is embedded in LSB of DWT coefficients of the image[10]. Steganographic approach to hide audio signal within the image using DWT proves to provide increased security [11]. The image is segmented region wise and is combined with PSO which results in SRG (Seeded Region Growing). Similarity criteria are solved by similarity difference between region value and pixel intensity [12].

Particle swarm optimization results in improved robustness with optimum results [13]. Unification factor is integrated with particle swarm optimization for balancing social terms and cognitive effects. Thus UPS (Unified Particle Swarm) is obtained by which particle is moved towards the center of global best and local best [14]. PSO is implemented by subtracting between personal best information and particle's current position. Distribution based update is proposed for particle swarm optimization [15].

Image classification procedure by extracting rotation invariant image features in SVD and DWT domain. Image texture classification is exploited by SVD [16], to find best pixel position to hide the secret image into cover image. It focuses on achieving acceptable robustness, quality and a stego image with distortion tolerance [17]. A technique based on detection of complex and smooth regions in a cover image and image interpolation. Reference pixel location is constructed by local image activity in a binary image. More reference pixels in the complex region is chosen, and embedding is done with fewer pixels to reduce image degradation [18]. Steganography technique with five layer protection is implemented for achieving high embedding capacity [19]. Contourlet transform and fresnelet transform are used for hiding data. To improve steganographic performance and to determine best coefficients GA and PSO are employed [20].

\section{Proposed Method}

Image steganography is achieved in two steps which are embedding and extraction. For embedding secret image and cover image are needed. In this method, cover image acts as stego key. The discrete wavelet transform is applied to cover image and required pixel for embedding in the secret image is selected using PSO. The secret message is embedded in an optimal position. Now IDWT is applied. The two major technique employed in this paper are given in Fig. 1.

\section{Particle Swarm Optimization}

The main goal of using PSO is to select optimal pixel position for embedding. In this approach pixel position is selected based on the cost function. The cost function is based on entropy, edge and seed point intensity. The calculation of all the above factors is based on the mean of particle adjacent seed points.

\section{DWT}

The next main goal is using DWT for representing the image in spatial domain which is into frequency domain with high and lowfrequency coefficients.

\subsection{Preprocessing}

Cover image act as stego key. The cover image is where the secret message is being embedded. Consider the cover image of size $\mathrm{P}^{*} \mathrm{Q}$. The cover image is generally in spatial domain, so DWT is applied to represent in the frequency domain with high and low-frequency coefficients. The cover image is subjected to DWT and subsampling to achieve better robustness. As a result, coefficients of the band obtained are

$$
\left[C_{1} C_{2} C_{3} C_{4}\right]=\operatorname{DWT}(\mathrm{C})
$$

Where $\mathrm{C} 1$ is lower frequency band coefficient which has all significant information about the image. C2,C3,C4 are insignificant wavelet coefficients which have information like an edge of image and texture. $\mathrm{C} 1$ and $\mathrm{C} 4$ bands are selected for further process. DWT is applied to $\mathrm{C} 1$ and $\mathrm{C} 4$, the coefficient of sub band extracted is

$\left[C_{1}^{L L} C_{1}^{L H} C_{1}^{H L} C_{1}^{H H}\right]=\operatorname{DWT}\left(C_{1}\right)$
$\left[C_{4}^{L L} C_{4}^{L H} C_{4}^{H L} C_{4}^{H H}\right]=\operatorname{DWT}\left(C_{4}\right)$

Where $C_{1}^{L L}$ is low-frequency sub bands of $C_{1}$ and $C_{4}^{L L}$ is lowfrequency sub bands of $C_{4}$. The remaining are high-frequency sub bands of $C_{1}$ and $C_{4}$ respectively.

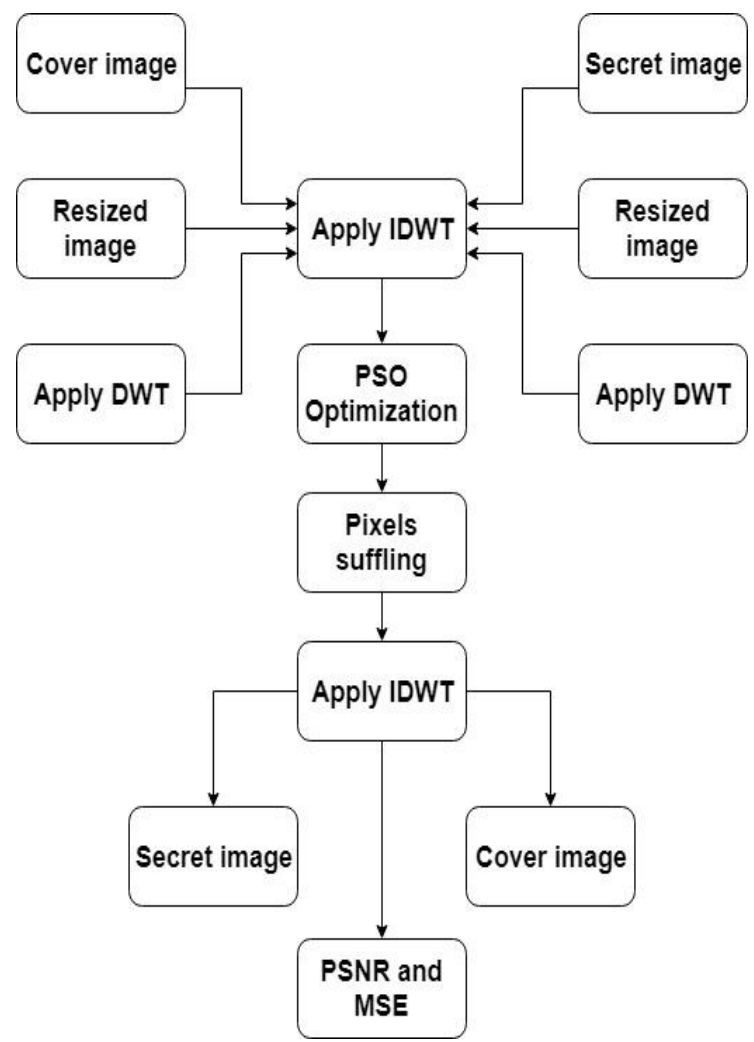

Fig. 1: Flow Chart of Proposed System

\subsection{Embedding}

Stego image is obtained when the secret message is embedded in to cover image. Statistical property of both stego image and cover image are similar. Embedding process is most efficient here because cover image acts as a stego key. Embedding process is as follows. First PSO is applied on the secret message to select an optimum position for embedding. Since DWT is applied and the subbands are in the frequency domain, IDWT is applied so that the image will be back in the spatial domain.

$C_{1}^{*}=\operatorname{IDWT}\left(C_{1}^{* L L} C_{1}^{* L H} C_{1}^{* H L} C_{1}^{* H H}\right)$

$C_{4}^{*}=\operatorname{IDWT}\left(C_{4}^{* L L} C_{4}^{* L H} C_{4}^{* H L} C_{4}^{* H H}\right)$

Modified bands $C_{4}$ and $C_{1}$ of embedding signal are

$C^{*}=\operatorname{IDWT}\left(C_{1}^{*} C_{2} C_{3} C_{4}^{*}\right)$

\subsection{Extraction}

In extraction process, a secret message has to be extracted from the cover image. DWT is applied to stego image for frequency representation of an image. In the extraction process, stego image is given as input. DWT is used for representing the image in spatial domain which is into frequency domain with high and lowfrequency coefficients. They decide various factors such as texture, edge, and significant details. Next step is extracting secret message by generating subbands.

\subsection{PSO Algorithm}

PSO algorithm is used to find pixel optimum position. The cost function is proposed to evaluate the fitness of pixel position. Cost function considers factors such as entropy, edge, and individual seed point edge. Initialization is performed by selecting the 
particles randomly and searching for an optimal solution with the help of updated position of all particles. Best identified position undergoes embedding. The following are the steps followed in PSO Step 1: Initialization

In dimensional size area $X^{*} Y$, let's consider $n$ no of populations which are given as $\mathrm{P}=\left\{P_{1}, P_{2}, \ldots, P_{n}\right\}$. Each population has some particles associated with them, initialize the position as well as velocities associated with the particle by random selection.

Step 2: Fitness evaluation

The fitness function is used to find a personal best position of particles. The fitness of all particles is determined. The fitness function is based on cost matrix.

Step 3: Determine the optimal solution

In population, the fitness value of all particles is evaluated by the fitness function. This search is done to find best position of particles and embedding is done

Step 4: Update the position

Entire search is done to find the optimal position and is updated. We consider a series of the previous iteration to find the optimal position

Step 5: Iteration

Repeat the above steps 2 to 4 times until you get optimal position for embedding.

\section{Experimental Results}

To illustrate the efficiency of the proposed system, some existing results are taken and compared regarding robustness and imperceptibility, the results have been compared. A fair comparison has been made using the case study with the related specification. MATLABR2014b is used to code the implementation and run on a PC with CPU Intel Pentium A1018 at $2.10 \mathrm{GHz}$. Image of size $512 * 512$ is taken, and the processing is done for gray scale images

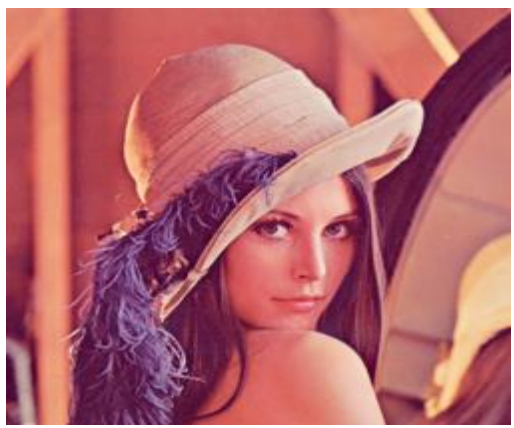

Fig. 2: Cover Image

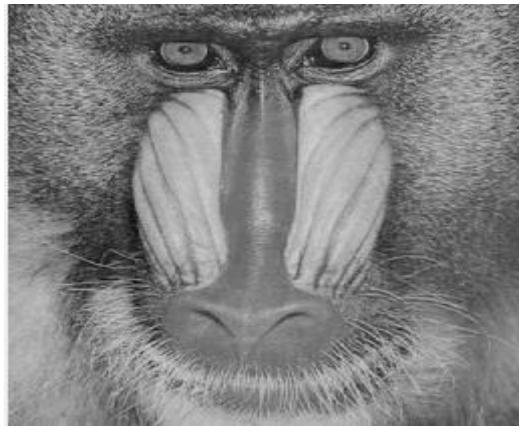

Fig. 3: Secret Image

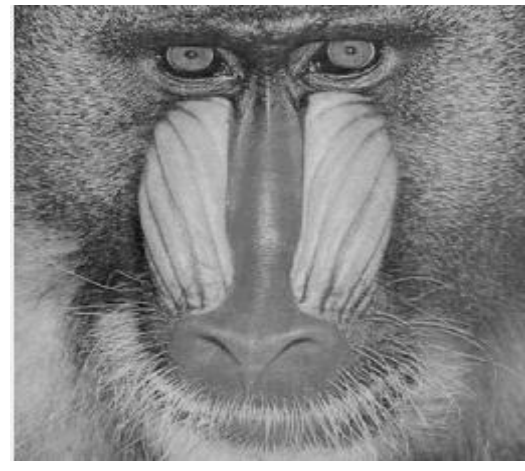

Fig. 4: Steganographed Image

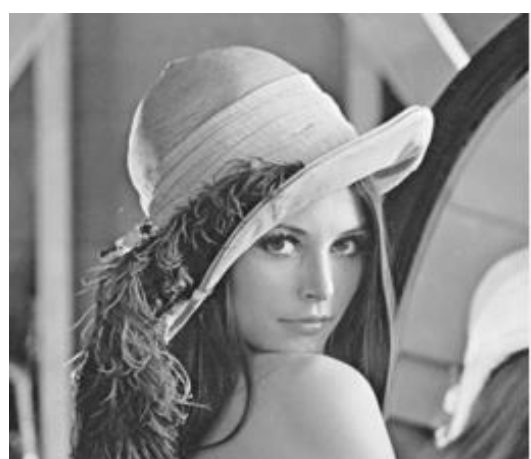

Fig. 5: Extracted secret image

In above images, Fig 2 represents the cover image. Fig 3 represents the secret image. Fig 4 represents the steganographed image. Fig 5 represents extracted the secret image. Quality of an image is measured by PSNR. Here PSNR is calculated between stego image and cover image. The following tabulation shows the comparison between [9] and the proposed technique.

\begin{tabular}{|c|c|c|}
\hline Image & {$[9]$} & Proposed scheme \\
\hline Lena & 41.3931 & 46.5431 \\
\hline Mandrill & 43.0865 & 48.1492 \\
\hline Peppers & 44.2146 & 50.0731 \\
\hline
\end{tabular}

MSE (Mean Error Square) determines image quality. It should be closer to zero. The following tabulation shows the comparison between [9] and the proposed technique.

\begin{tabular}{|c|c|c|}
\hline Image & {$[9]$} & Proposed scheme \\
\hline Lena & 0.9134 & 0.7643 \\
\hline Mandrill & 0.9058 & 0.6982 \\
\hline Peppers & 0.8012 & 0.6052 \\
\hline
\end{tabular}

\section{Conclusion}

The goal of image steganography is to improve the security and to embed the stego image in to cover image in such a way that it does not arise any suspicion among intruders. This technique proposed above tries to achieve better capacity, imperceptibility, and robustness. The main goal of using PSO is to select optimal pixel position for embedding. In this approach, pixel position is selected based on the cost function. The cost function is based on entropy, edge and seed point intensity. The next main goal was using DWT for representing the image in spatial domain which is in the frequency domain with high and low-frequency coefficients. The PSNR and MSE values obtained prove that the proposed technique is comparatively better than the existing techniques. 


\section{References}

[1] S. K. Sabnis and R. N. Awale, "Statistical Steganalysis of High Capacity Image Steganography with Cryptography," Procedia Comput. Sci., vol. 79, pp. 321-327, 2016.

[2] P. Sethi and V. Kapoor, "A Proposed Novel Architecture for Information Hiding in Image Steganography by Using Genetic Algorithm and Cryptography," Procedia Comput. Sci., vol. 87, pp. 61-66, 2016.

[3] G. Swain, "Digital Image Steganography Using Variable Length Group of Bits Substitution," Procedia Comput. Sci., vol. 85, no. Cms, pp. 31-38, 2016.

[4] M. S. Subhedar and V. H. Mankar, "Image steganography using redundant discrete wavelet transform and QR factorization," Comput. Electr. Eng., vol. 54, pp. 406-422, 2016.

[5] M. Taherkhani and R. Safabakhsh, "A novel particle swarm optimization algorithm with adaptive inertia weight," Appl. Soft Comput., vol. 38, pp. 281-295, 2016.

[6] K. Ahmadi, A. Y. Javaid, and E. Salari, "An efficient compression scheme based on adaptive thresholding in wavelet domain using particle swarm optimization," Signal Process. Image Commun., vol. 32, pp. 33-39, 2015.

[7] R. Bhardwaj and V. Sharma, "Image Steganography Based on Complemented Message and Inverted Bit LSB Substitution," Procedia Comput. Sci., vol. 93, no. September, pp. 832-838, 2016.

[8] M. Jain, S. K. Lenka, and S. K. Vasistha, "Adaptive circular queue image steganography with RSA cryptosystem," Perspect. Sci. vol. 8, pp. 417-420, 2016.

[9] M. Tang, S. Zeng, X. Chen, J. Hu, and Y. Du, “An adaptive image steganography using AMBTC compression and interpolation technique," Optik (Stuttg)., vol. 127, no. 1, pp. 471-477, 2016.

[10] U. Dewangan, M. Sharma, and S. Bera, "Development and Analysis of Stego Image Using Discrete Wavelet Transform," vol. 2, no. 1, 2013.

[11] S. Hemalatha, U. D. Acharya, and A. Renuka, "Wavelet transform based steganography technique to hide audio signals in image," Procedia Comput. Sci., vol. 47, no. C, pp. 272-281, 2014.

[12] F. Mohsen, M. Hadhoud, K. Mostafa, and K. Amin, "A New Image Segmentation Method Based on Particle Swarm Optimization," Int. Arab J. Inf. Technol., vol. 9, no. 5, pp. 487493, 2012.

[13] S. Gayathri and D. Venkatesan, "Particle Swarm Optimization and Discrete Wavelet Transform based Robust Image Watermarking," Indian J. Sci. Technol., vol. 9, no. 48, 2016.

[14] H. C. Tsai, "Unified particle swarm delivers high efficiency to particle swarm optimization," Appl. Soft Comput. J., vol. 55, pp. 371-383, 2017.

[15] M. S. Kiran, "Particle swarm optimization with a new update mechanism,” Appl. Soft Comput. J., vol. 60, pp. 670-678, 2017.

[16] B. M. Chang, H. H. Tsai, and C. Y. Yen, "SVM-PSO based rotation-invariant image texture classification in SVD and DWT domains,” Eng. Appl. Artif. Intell., vol. 52, pp. 96-107, 2016.

[17] P. Bedi, R. Bansal, and P. Sehgal, "Using PSO in a spatial domain based image hiding scheme with distortion tolerance," Comput. Electr. Eng., vol. 39, no. 2, pp. 640-654, 2013.

[18] W. Hong and T. S. Chen, "Reversible data embedding for high quality images using interpolation and reference pixel distribution mechanism," J. Vis. Commun. Image Represent., vol. 22, no. 2, pp. 131-140, 2011.

[19] N. N. El-Emam, "New data-hiding algorithm based on adaptive neural networks with modified particle swarm optimization," Comput. Secur., vol. 55, pp. 21-45, 2015.

[20] S. Uma Maheswari and D. Jude Hemanth, "Performance enhanced image steganography systems using transforms and optimization techniques," Multimed. Tools Appl., vol. 76, no. 1, pp. 415-436, 2017.

[21] T. Padmapriya, V.Saminadan, "Performance Improvement in long term Evolution-advanced network using multiple imput multiple output technique", Journal of Advanced Research in Dynamical and Control Systems, Vol. 9, Sp-6, pp: 990-1010, 2017.

[22] S.V.Manikanthan and K.Baskaran "Low Cost VLSI Design Implementation of Sorting Network for ACSFD in Wireless Sensor Network", CiiT International Journal of Programmable Device Circuits and Systems, Print: ISSN 0974 - 973X \& Online: ISSN 0974 - 9624, Issue: November 2011, PDCS112011008. 